\title{
Generation of Predictive Price and Trading Volume Patterns in a Model of Dynamically Evolving Free Market Supply and Demand
}

\author{
J. K. WANG* \\ Pinetree Management, Inc., 460West Mountain Road, Sparta NJ 07871
}

(Received 29 May 2000)

\begin{abstract}
I present a model of stock market price fluctuations incorporating effects of share supply as a history-dependent function of previous purchases and share demand as a function of price deviation from moving averages. Price charts generated show intervals of oscillations switching amplitude and frequency suddenly in time, forming price and trading volume patterns well-known in market technical analysis. Ultimate price trends agree with traditional predictions for specific patterns. The consideration of dynamically evolving supply and demand in this model resolves the apparent contradiction with the Efficient Market Hypothesis: perceptions of imprecise equity values by a world of investors evolve over non-negligible periods of time, with dependence on price history.
\end{abstract}

Keywords and Phrases: Discrete tatonnement; Efficient Market Hypothesis; Market pricing; Predictive price patterns; Supply and demand

\section{INTRODUCTION}

A fundamental tenet underlying chart technical analysis is the belief that future changes in prices of market securities are predictable, within a range of precision, based on the prior history of traded prices and volume (number of shares traded in one time period). Extensive literature exists detailing different methods of technical analysis; see for example Pring (1991) and Edwards and Magee (1992). Numbers of chart technicians and their organizations past and present have traded profitably using these methods. Chart technical analysis has traditionally not been seriously regarded by academic researchers, however, due to the apparent paradox that if all future prices are foreseeable due to well-known techniques, then trades by analysts must always be too late to profit from predictions (an application of the Efficient Market Hypothesis). Market technicians, behavioral economists, and presumably all discretionary investors and analysts, resolve this paradox as due to slow

*Tel.: (973) 726-0910, Fax: (973) 726-0910, e-mail: pinetreescsw@worldnet.att.net 
evolution of perceptions of imprecise value by a diverse spectrum of investors, resulting in and influenced by dynamically evolving strengths of supply and demand for shares.

In technical analysis, concepts such as price levels of support and resistance and sentiment are used in making detailed analyses and projections of supply and demand levels. Price levels define ranges of predicted price movements for traded securities and indices. Sentiment refers to quantitative measures of the dominant aggregate tendency of investors to buy or to sell. At present, however, no complete explicit mathematical model exists of the interplay of investor actions, psychology, and prices to test and analyze consistency of these concepts. That is, although an extensive body of analytical techniques exists for predicting future behavior of markets, a mathematical model remains to be developed that reproduces realistic market behavior, giving a picture of the basic mechanisms underlying free market dynamics.

Day (1997) has recently developed a model of market price action in the absence of information shocks that takes into account investor supply and demand and interactions due to market mediation (by a specialist or marketmaker). Prices generated by his model exhibit chaotic, quasi-periodic behavior-irregular oscillations which do not follow a simple regular formula - and patterns similar to well-known formations from technical analysis. Wang (1998) has noted, however, that in Day's model share supply and demand, price jumps and volume, are all functions independent of time except through dependence on the last traded price. This is in contradiction with observations in reallife markets and with the principle from technical analysis of ever-changing supply and demand underlying price resistance and support levels.

In this paper I extend Day's model to include: share supply (sell orders) as a dynamic function dependent on the history of previous purchases; share demand as a function of the current price relative to moving averages; and trading volume including the specialist's contribution. The mathematical formulation and some preliminary discussion of the dynamically evolving model are presented in Sections II and III below. In Section IV I present results of calculations using the model, including theoretical price charts generated by the model. I find that using a wide range of parameters, quasi-periodic price behavior also occurs in this model with evolving supply and demand. I examine parameters representing various supply and demand scenarios, bullish, bottoming, topping, and compare results to frequently observed real stock price patterns. In Section V I conclude with discussion of limitations and assumptions in the model and suggestions for further study and improvements.

\section{MATHEMATICAL FORMULATION OF THE MODEL}

As discussed in the Introduction, I developed my model beginning with several assumptions from Day's market price model. First, I assume trading by two types of investors, value/contrarian $(A)$ and short-term/trader $(B)$, and by a marketmaker or specialist who adjusts prices in a discrete tatonnement process dependent on the supply/demand imbalance for shares of stock. Throughout the rest of this paper, this model will be discussed in relation to "stock" prices and shares; all equations (with changes of parameters) should apply to any security, index, commodity or derivative traded in an open market. Second, I assume that, at any specific time $t$ and current trading price $P(t)$, the price $P(t+1)$ in the next period (e.g., hour, day, week) shifts by an amount proportional to excess total market supply of shares $\left(S_{\text {tot }}\right)$ over total market demand for shares $\left(D_{\text {tot }}\right)$; so that

$$
P(t+1)=P(t)\left[1+C_{t}\left(D_{\mathrm{tot}}-S_{\mathrm{tot}}\right)\right],
$$

where $C_{t}$ is a constant parameter for this tatonnement process.

In addition I model the corresponding trading volume $V(t)$ for study as well, volume being nearly as important a variable as price itself in technical analysis. Share volume traded at each $P(t)$ of 
course includes all investor share supply and demand which can be directly matched. At each time $t \mathrm{I}$ also assume a constant fraction $c_{\mathrm{sp}}$ of remaining supply or demand imbalance is traded at $P(t)$ by the specialist in the process of adjusting price to $P(t+1)$. That is,

$$
\begin{aligned}
V(t)= & \left(1-c_{\mathrm{sp}}\right) \min \left(D_{\mathrm{tot}}, S_{\mathrm{tot}}\right) \\
& +c_{\mathrm{sp}} \max \left(D_{\mathrm{tot}}, S_{\mathrm{tot}}\right) .
\end{aligned}
$$

On the NASDAQ or other exchanges without specialists, the behavior of the equivalent marketmaker is presumably similar, with different value of $c_{\mathrm{sp}}$ (perhaps 0 ).

Total supply of/demand for stock shares is simply equal to the sum of supply/demand from the different investor types:

$$
S_{\mathrm{tot}}=S_{A}+S_{B}
$$

and

$$
D_{\text {tot }}=D_{A}+D_{B}
$$

For common stocks, values are not precisely known and are generally perceived as different by different investors. Share supply and (indirectly) demand can then both depend to large extent upon trading history. Stock buying is primarily pricedriven, by expectation of selling in the future at a higher price. In the absence of precise measures, investors must estimate stock purchase value relative to the range of past traded prices, i.e., by how much the market is "going up". If shorting of unowned shares comprises relatively little of total share supply, then selling is by previous buyers seeking gain (or minimal loss), also relative to the prices at time of original purchase. It is noted that market trading history is important even to investors who value using price/earnings ratios or other "fundamental" indicators, since they ultimately evaluate numbers relative to ranges of historically observed values.

Following the above arguments $S_{i}$ and $D_{i}, i=A$ and $B$, were modeled to have dependence on (a) the evolving history of past trading prices, $P(s$; $s \leq t$ ), and (b) the distribution of shares currently owned at time $t$ and originally purchased at prices $p$, summarized as "ownership" functions $N_{i}(p, t)$ (ignoring dividends). The evolving dependence (a) on past prices is approximated as dependence on moving averages of past prices $P_{i \text { avg }}(t)$. Movingaverages are widely used in technical analysis to determine optimal purchase and sale prices; see for example Pring (1991); Edwards and Magee (1992); and Ross (1998). Assuming that analysts do not themselves dominate market behavior, the success of such indicators is evidence that investor supply and demand in real markets do have strong dependence on price moving-averages. The model "memory" variable $N_{i}(p, t)$ (b) includes no information on times of earlier purchases at prices $p$. This implies that length of ownership has no effect on sale price. This is not strictly true in technical analysis but is a reasonable approximation; see Pring (1991), p. 186. If shorting except by the specialist is ignored, then $N_{i}(p, t)$ is always positive.

For each investor type $i$, functions $S_{i}$ and $D_{i}$ can assume different functional dependences on price history and different parameter values. The functions assumed in this paper are:

$$
\begin{aligned}
& D_{A}[P(t)]=C_{A} \max \left\{1-P(t) / P_{A} \text { avg }(t), 0\right\}^{n}, \\
& D_{B}[P(t)]=C_{B} L_{B} \tanh \left[\mid 1-P(t) / P_{B} \text { avg }(t) \mid / L_{B}\right], \\
& S_{i}[P(t)]=\sum s_{i}[P(t), p], \quad \text { summed over } p,
\end{aligned}
$$

where

$$
s_{i}[P(t), p]=R N_{i}(p, t) \tanh ^{n}\left[|1-P(t) / p| / L_{i}\right],
$$

and

$$
\begin{aligned}
& N_{i}(p, t)=N_{i}(p, t-1)-s_{i}[P(t-1), p] \\
& \min \left\{V(t-1) / S_{\text {tot }}[P(t-1)], 1\right\} \\
& +\quad[\text { if } p=P(t-1)] \\
& \min \left\{V(t) / D_{\text {tot }}[P(t-1)], 1\right\} \\
& D_{i}[P(t-1)] \text {. }
\end{aligned}
$$


$C_{i}, R, L_{i}$ and $n$ in the above equations are all constant parameters. As discussed in the preceding paragraph, $P_{i}$ avg $(t)$ in Eqs. (4) are exponential moving averages of $P(t)$, with fixed time constant parameters. Investor $B$ demand [Eq. (4b)] in this model combines demand functions of short-term value (buying on small dips) and short-term trend (buying on small jumps) investors. As price deviations increase from zero, the tanh dependences in Eqs. (4b) and (5b) increase asymptotically from power-law dependences to constants. Intuitively, supply and demand for a given stock cannot grow without limit in a finite market system.

\section{COMMENTS ON THE MODEL}

At each point in time all model variables in the model are calculated based iteratively on values one time interval before. At time 0 I start with assumed initial values for $P(0), P_{i \text { avg }}(0)$, and $N_{i}(p$, $0)$. Equations (4) and (5) determine functions $S_{i}$ and $D_{i}$, from which Eqs. (1) and (2) generate $V(0)$, $P(1)$ and $P_{i \text { avg }}(t)$. Equation (6) then generates $N_{i}(p, 1)$. The routine repeats, using values at time $t$ to generate values at time $t+1$. Due to limits on computational power, ownership functions $N_{i}(p, t)$ were approximated in actual calculations as total stock purchased at prices within unit intervals of $p$ rather than at actual prices $p$ (i.e., as discrete rather than continuous functions). For reasonably large values of $p$, this approximation did not qualitatively affect results. Also due to computational limits, just 3 investor types were modeled in Section II: long-term value investor, short-term buyer-on-dips, and short-term buyerof-trends. While diversity is no doubt much greater in real markets, it was hoped that important features of real markets would still appear in this model approximation.

Some comments are in order regarding the constant parameters in Eqs. (4) and (5): $C_{i}, R, L_{i}$ and $n$, as well as the time constants for moving averages $P_{i \text { avg }}(t)$.
- In Eqs. (4) $C_{i}$ are constant factors determining size of investor demand. They give a measure of interest and/or available liquidity from the different investor classes.

- In Eq. (5b), $R$ is similarly a constant factor modifying the actual size of supply, effectively defining the fraction of underlying supply appearing as actual market sell orders in each given time interval. $R$ is therefore a measure of negative sentiment or overall investor readiness to sell.

- In Eqs. (4b) and (5b), $L_{i}$ is a profit-stop parameter determining the range of price movement significant to investor $i . L_{B}$ appears in Eq. (4b) as a natural limit on demand, since investor $B$ presumably will not buy if prices have jumped from his/her perceived base price $P_{i \text { avg }}(t)$ by more than his/her expected profit amount. $L_{i}$ is thus a measure of the strength of investor conviction; i.e., of his/her risk tolerance for fluctuations in investment value.

- In Eqs. (4a) and (5b), variable $n$ is a constant exponent determining supply/demand price elasticity, or how "abruptly" supply/demand increases as prices approach profit-stop levels. $n$ should be larger for investor classes with welldefined trading strategies and for more homogeneous investor composition (e.g., for fewer but larger institutional investors rather than numerous smaller investors with a broad spectrum of trading strategies). For investor $B, n$ equals one, assuming that early response is characteristic of buying short-term price moves. To reduce calculational complexity, other investor types are assumed to have the same value of $n$ in a given price chart projection.

In a given price chart projection, precise values of the constant parameters discussed above are not so meaningful as the effects of variations of values in different price chart projections. A trivial example is that price behavior is unchanged if total number of stock shares were expressed in thousands or millions and all parameters were scaled accordingly. 


\section{RESULTS OF MODEL CALCULATIONS}

\section{IV.1. Head-and-Shoulders Pattern}

Figure 1 shows example supply and demand functions for investor types $A$ and $B$, assuming original purchases of 18.5 shares at 44.5 by $A$ and 5.5 shares at 45.5 and 46.5 each by $B$. For the sake of clarity supply functions are shown as negative. As noted above, number of shares in this model could be interpreted as millions of shares of a real exchange-traded stock. Initial moving-average prices are 46 for $A$ and $B$, with averaging time constants of 108 and 28 , respectively. Other parameters are: $C_{A}=240, C_{B}=14, R=9 \%, L_{A}=$ $16 \% L_{B}=6 \%$, and $n=3.5$. Table I summarizes all initial and constant parameters used for price projection studies in this section. Following Eq. (4a) long-term value investor $(A)$ demand [solid dark line] occurs only for prices below the long-term moving-average. Price dependence is highly non-linear; value investor $A$ buys only at prices well below his perceived base average.
Short-term investor $(B)$ demand [Eq. (4b), solid light line] increases with both positive and negative price fluctuations around the moving-average price. Price dependence is linear for small price fluctuations but asymptotically constant for larger fluctuations, as discussed in Section III.

Both long- and short-term investor supply functions [Eq. (5b), line with markers and dashed line] are symmetric about the initial purchase prices, proportional to number of shares purchased. Supply functions are highly non-linear $(n=3.5)$ so that significant supply appears only after significant price movement has occurred after initial purchases. Like demand, supply levels off at extreme price deviations. With the given parameters, supply is initially smaller than demand in Figure 1; prices therefore rise in near-future time periods (Fig. 2). After more shares have traded at increasing prices, supply increases at all prices. Simultaneously, price moving-averages will converge towards the trading price range. Investor $A$ demand decreases as prices rise; $B$ demand does not decrease as much due to $B$ 's shorter time constant. The resulting price charts will show

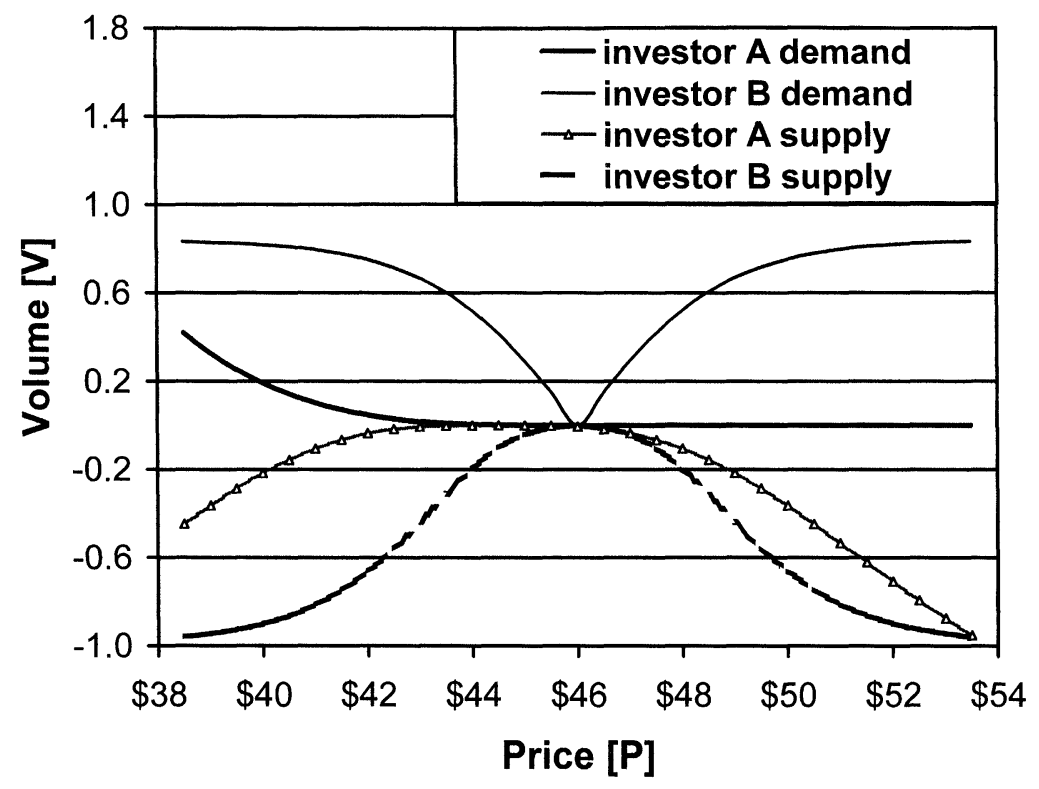

FIGURE 1 Supply and demand functions [volume $V$ (number of shares) versus price $P$ ] at $t=0$ based on Eqs. (4) $-(5)$ and indicated initial parameters in Table I generating Head-and-Shoulders price pattern. 
TABLE I Initial parameters for various chart patterns

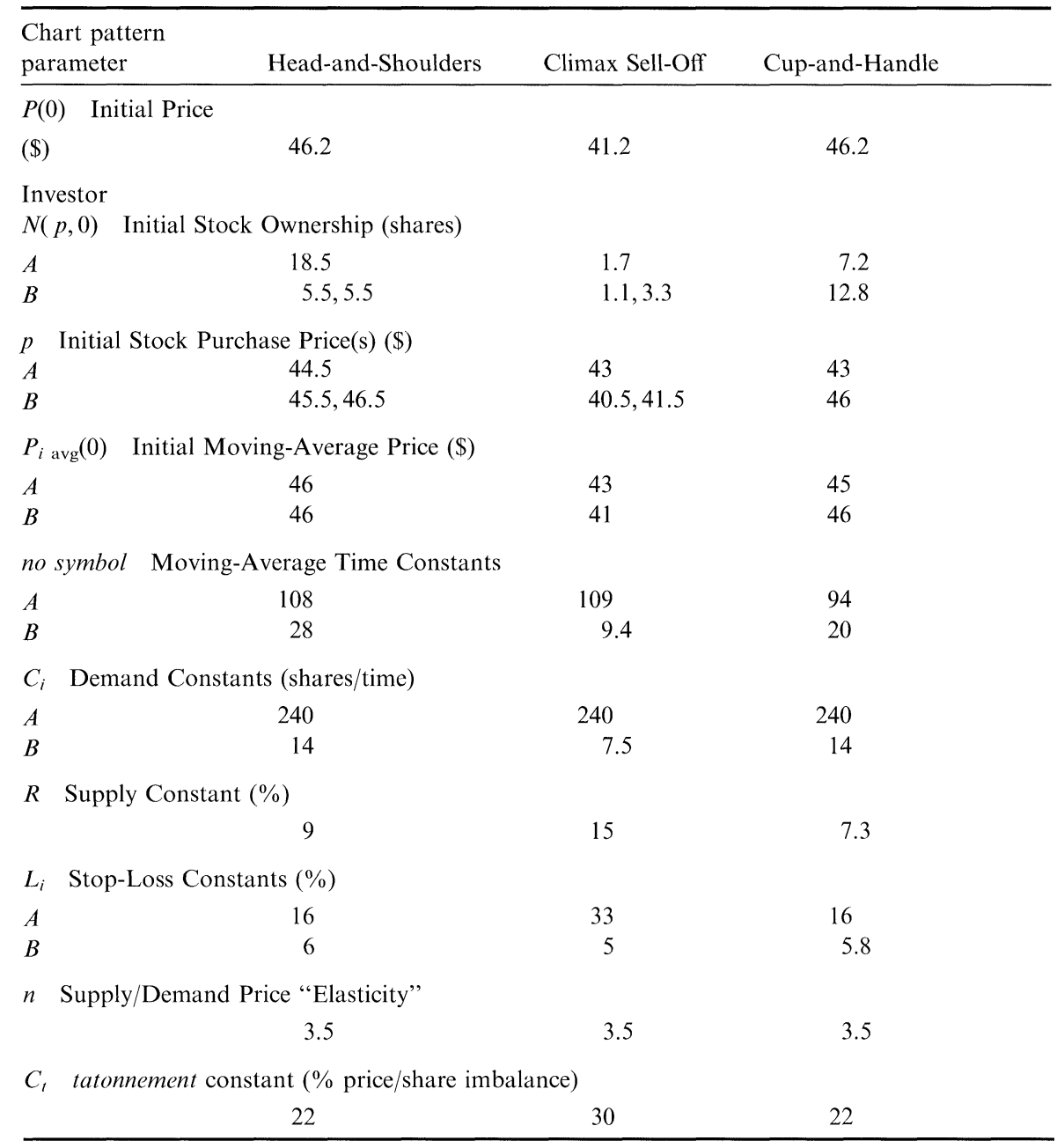

rising prices until supply exceeds demand. More complex behavior results thereafter, dependent upon the interplay of supply and demand from the two investor types.

Figure 2 shows the complete resulting price/ volume chart calculated from the model equations in Section II, using the initial parameters specified for Figure 1. $C_{t}=22 \%$ for this example. The chart reproduces the classic head-and-shoulders formation from chart technical analysis and the expected price drops following. Consistent with heuristic arguments of technical analysis, volume on the left shoulder is primarily investor $A$ selling to investor
$B$, as $A$ 's profit-stop objective is approached (slightly below $116 \% \times 44.5=51.12$ ). Volume on the right shoulder $[t>65]$ is lighter, arising from "late" $B$ investor buying and none by $A$ investors. The left shoulder is not as well-developed as the right in this particular calculation. Price curve oscillations are smoother than usually observed in daily and weekly charts, more characteristic of intraday price charts. Smoothness is consistent with stock price movement over shorter periods of time, where the period-by-period model equations and parameters in Section II would be expected to hold most accurate. Reproduction of the irregular 


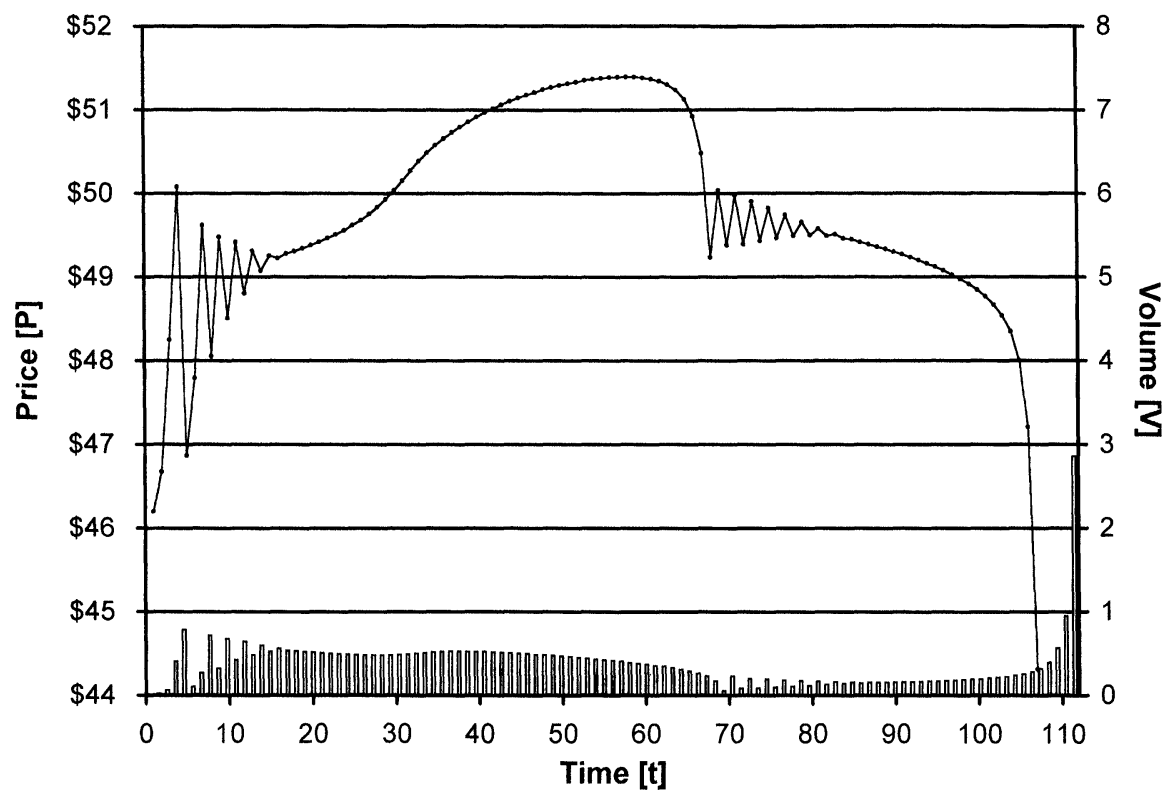

FIGURE 2 Head-and-Shoulders price pattern [price $P$ and volume $V$ versus time $t$ ] generated from Eqs. (1)-(5) and indicated initial parameters in Table I.

backing and filling cycles associated with longerterm charts may require more computational power and possibly additional parameters (see also discussion below and Wang (preprint)). Approximate calculations generated a price rebound, a retracement expected in technical analysis, after the fall from the right shoulder. Due to limits on computational power, however, prices were not precisely projected below 44 in Figure 2.

Similar head-and-shoulders patterns were also generated (not shown) with other sets of initial parameters: for example, by increasing initial $A$ shares to $20.0, n$ to 4.2 , and $R$ to $11 \%$. The pattern generated by this variation extended over a longer period of time. An intuitive explanation is as follows: increasing initial shares and $R$ effectively expands share supply and trading activity which must equilibrate over longer time in developing into a head-and-shoulders; increasing $n$ extends the price range and time before sentiment responds to price changes. In general, however, patterns change significantly with arbitrary variations of initial parameters. Section IV.2 below provides an illustration.

\section{IV.2. Climax Sell-Off Pattern}

Figure 3 shows supply and demand functions for investor types $A$ and $B$, assuming original purchases of 1.7 shares at 43 by $A$ and 1.1 shares at 40.5 and 3.3 shares at 41.5 each by $B$. For $A$ and $B$ respectively, initial moving-average prices are 43 and 41; time constants are 109 and 9.4. Other parameters are: $C_{A}=240, C_{B}=7.5, R=15 \%, L_{A}$ $=33 \%, L_{B}=5 \%$ and $n=3.5$ [see Tab. I]. Supply functions are again negative-valued as in Figure 1. Parameter $C_{A}$ and exponent $(n=3.5)$ of price dependence are the same as in Figure 1, but unit price changes are a larger fraction of the type $A$ moving-average; value investor demand [solid dark line] therefore is larger than in Figure 1. Short-term investor $(B)$ demand [solid light line] has the same functional form as in Figure 1, but is smaller, due to smaller $C_{B}$ and $L_{B}$. Long-term supply [line with markers] is much smaller than in Figure 1 due to larger $L_{A}$; short-term investor supply [dashed line] is comparable in magnitude to $L_{B}$ in Figure 1. With the given parameters supply is again initially smaller than demand, and prices 


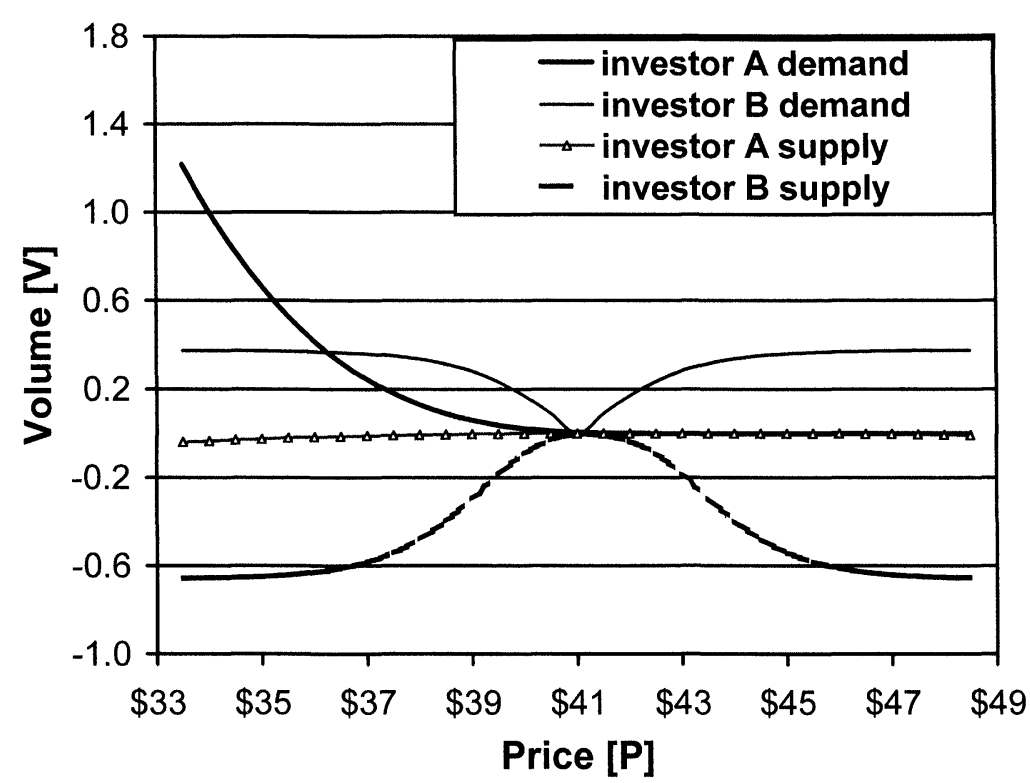

FIGURE 3 Supply and demand functions [volume $V$ (number of shares) versus price $P$ ] at $t=0$ based on Eqs. (4) - (5) and indicated initial parameters in Table I generating Climax Sell-Off price pattern.

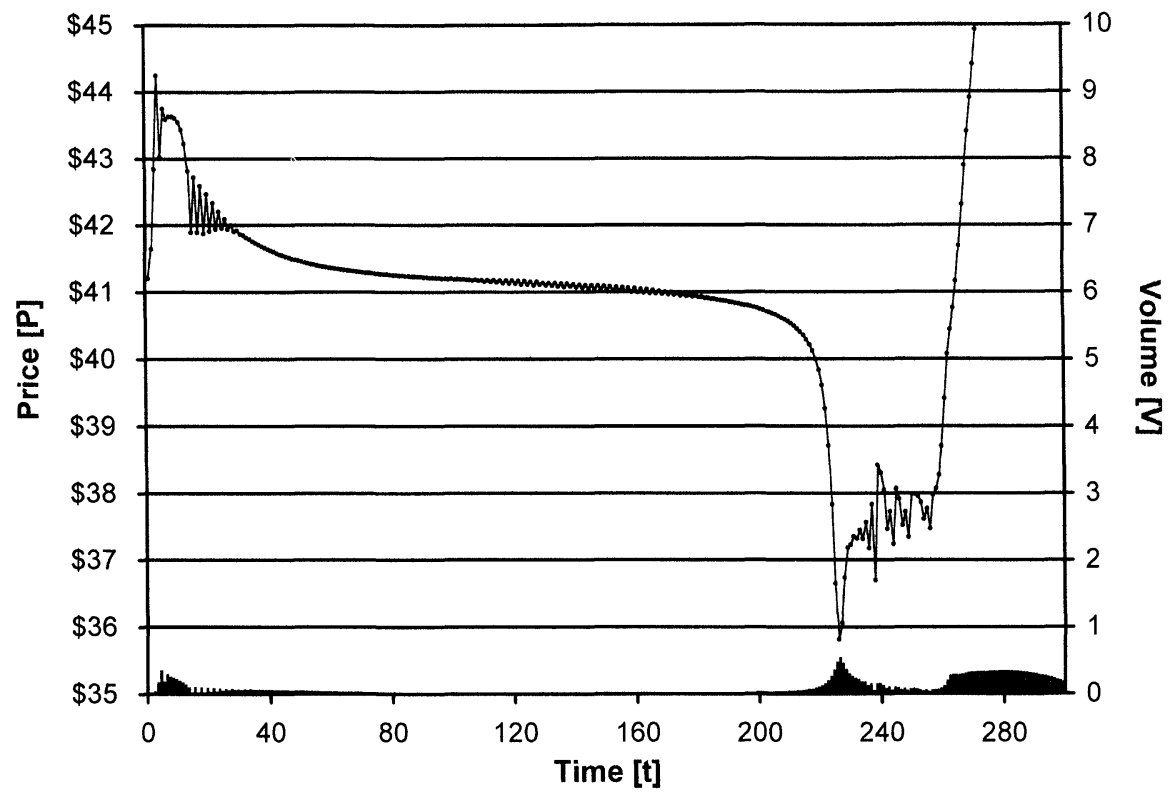

FIGURE 4 Sell-Off price pattern [price $P$ and volume $V$ versus time $t$ ] generated from Eqs. (1) - (5) and indicated initial parameters in Table I.

rise in near-future time periods. In comparison with Section IV.1, investor $B$ is much more a short-term trader, given that $C_{B}, L_{B}$, and investor $B$ time constant are all smaller.
Figure 4 shows the resulting price/volume chart calculated from the model equations in Section II, using the initial parameters specified for Figure 3. $C_{t}=30 \%$ for this example. A very realistic climax 
sell-off scenario is generated, followed by clear change to bullish trend. Generation of this scenario is again consistent with arguments of technical analysis. Overall sentiment is bearish, characterized by relatively large $R$ and presence of only short-term traders and "strong hands" value investors (large $L_{A}$ ). But total number of shares trading by $A$ and $B(1.7+3.3+1.1=6.1)$ is lower than in Section IV.1, so that long-term fundamentals are more bullish. Early price advances are not sustained. A final ascending-triangle bottom, a familiar oscillatory pattern from technical analysis, allows shares to be accumulated by "strong hands" and a bullish trend is established. As in real price charts, volume is high on the initial sharp sell-off, but less on successive price drops. A slight inflection is visible in the price chart where prices rise through initial levels [time $(t) \sim 260]$. Absence of more congestion at this price level, and the smoothness of the price curve, suggests that Figure 4, like Figure 2, is more characteristic of shorter-term price action with shorter times for development of support and resistance levels.

\section{IV.3. Cup-and-Handle Pattern}

Figure 5 shows a third and final price chart. Supply and demand functions are not shown for this example. Original purchases are 7.2 shares at 43 by $A$ and 12.8 shares at 46 by $B$. For $A$ and $B$ respectively, initial moving-average prices are 45 and 46; time constants are 94 and 20. Other parameters are: $C_{A}=240, C_{B}=14, R=7.3 \%$, $L_{A}=16 \%, L_{B}=5.8 \%$ and $n=3.5$. Several parameters and exponent $n(=3.5)$ of price dependence are the same as for Figures 1 and 2; $R$ and the time constant for investor $A$ are smaller. Total number of shares (20) is between the totals for the preceding two scenarios in Sections IV. 1 and IV.2, implying a neutral to moderately bullish market environment given that several other parameters are the same.

The actual price/volume chart calculation in Figure 5 confirms the bullish environment and reproduces the cup-and-handle pattern discussed extensively by O'Neil (1995) in connection with bullish stock situations. $C_{t}=22 \%$ for this example. After an initial jump of prices upward, prices

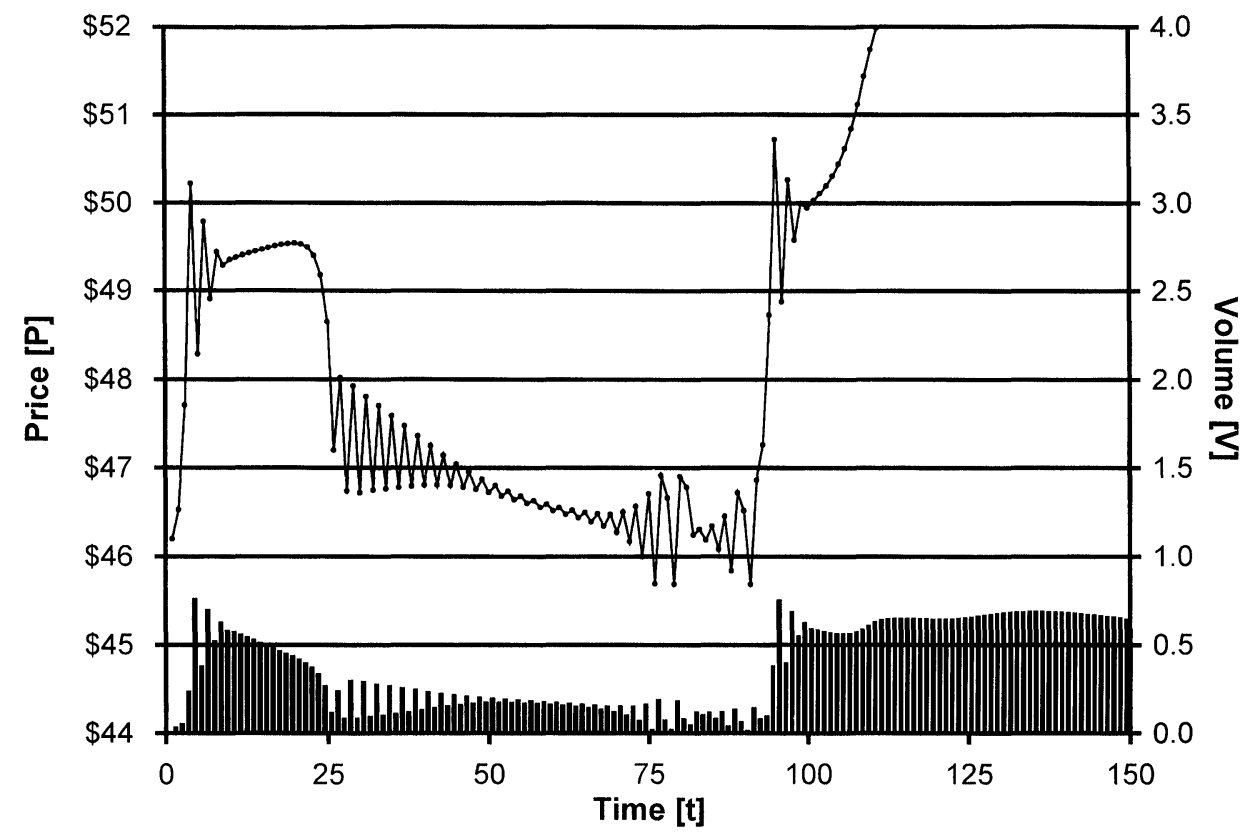

FIGURE 5 Cup-and-Handle price pattern [price $P$ and volume $V$ versus time $t$ ] generated from Eqs. (1)-(5) and indicated initial parameters in Table I. 
settle between $\$ 49$ and $\$ 50$, then settle back down on lower volume into a rounded cup- or saucershaped formation. After a few irregular tests of the bottom (around $\$ 46$ ), prices retest the initial high at $\$ 50$, settle in the cup handle just above, then climb further upward. Model calculation results indicate that $A$ investors sell almost all of their shares by the end of the breakout from the pattern handle; most selling is at the $\$ 50$ resistance level. Computational limitations prevented longer-term study of the development of this scenario. Presumably, parameters eventually change over very long periods of time; or very long-term investors (including insiders and a company itself through secondary offerings and buybacks) may begin to distribute their shares after an extended bullish period.

\section{DISCUSSION OF RESULTS}

The results of this paper demonstrate that realistic market price patterns are reproduced within a consistent mathematical model [Eqs. (1)-(5)] based on principles of technical analysis. Arbitrary changes in initial parameters lead to different patterns, confirming that certain patterns in technical analysis have strong significance and imply specific market conditions. Sensitivity to initial parameters, ostensibly lack of robustness, actually confirms the implicit uncertainty associated with the "art" of chart analysis. Certain price activity may strongly indicate bearishness, for example, but the exact time and price of the "top" can only be projected with some uncertainty. Different combinations of parameters can generate the same patterns on different time scales. This is consistent with the usefulness of chart technical analysis on many different time scales. It should be noted that the functional forms of supply and demand in Eqs. (4b) and (5b), respectively, were assumed solely for simplicity of expression. If functional expressions with similar asymptotic behavior were substituted (e.g., $2\left(\tan ^{-1} \pi x / 2\right) / \pi$ instead of $\left.\tanh x\right)$, the qualitative results generated in Section IV are expected to be reproducible with appropriate modifications of various constant parameter values. Results of variations in Eqs. (4)-(6), and interpretation in terms of investor behavioral characteristics, are discussed in another paper by Wang (preprint).

As mentioned in the Introduction, the model equations are elaborated from equations studied by Day (1997), with which he modeled market price response to excess supply/demand and resulting support/resistance levels. With its constant supply and demand functions Day's model is appropriate only over very short time intervals and when volume is small compared to total stock supply. The present paper incorporates effects on investor sentiment of trading history and limited stock supply into the model, resulting in dynamically evolving and interacting supply and demand and support/resistance levels. Price jumps, support/resistance levels, and volume now all depend on past prices and trading volume in a complex, non-reproducing manner. Relatively straightforward model equations and variables generating these results provide a connection between empirical observations of technical analysts and general principles proposed by behavioral economists. Functions and parameters are quantified defining sentiment, share supply, and investor character. Resulting price and volume charts allow study of how these characteristics interact in markets.

Several thoughts arise regarding future study of this model. In Section IV I have attributed unrealistic smoothness of price and volume in chart calculations as due to limits in computational detail; support and resistance levels are not well enough defined. As noted in earlier paragraphs, results have been obtained by Wang (preprint) implying that realistic price fluctuations can be associated with deterministic variations in the supply functions. The smoothness also suggests the intuition that timing of real trades depends probabilistically on supply and demand functions. That is, given supply and demand functions calculated in Eqs. (3)-(6), trading 
volume also depends on some stochastic factor multiplied times supply/demand. Such a formulation would be reminiscent of quantum statistical models of materials, where electrons within a crystal react and change states depending both on probability and on fixed material properties.

Extensive work remains in order to develop understanding of very long-term free market price dynamics. In Figures 2, 4 and 5, prices diverge either up or down beyond chart boundaries; other parameter assumptions (not discussed) resulted in convergence towards finite constant values. Such price behavior is consistent with that observed over intermediate but not very long time scales in real markets. For most real market securities, different chart formations eventually occur over the extended price history, all of which must be associated with different sets of parameters. What are the equations determining long-term transformations between "constant" parameter sets in terms of price and volume history? What are the global parameters and equations governing flow of money or investor demand among different stocks or sectors, leading to sector rotation, relative strength and correlations?

In closing, I note that this paper has focused on the validity of technical analysis based on classical chart formations. For the future, more recently developed chart techniques, such as MACD, stochastics, point-and-figure, Gann theory, should be evaluated in conjunction with this model. Are there relationships governing the model parameters that systematically generate Fibonacci series ratios commonly identified in longer-term charts? For more thorough analysis of price patterns, the current model also must be extended to generate bar (and candlestick) charts, involving a succession of time intervals separated by closed market periods. A related issue would then be how to define price gaps in chart calculations. It would also be of interest to incorporate bid/ask spreads and pricing by discrete fractions in the model. Clearly, the model presented is at an early stage of development and can be further improved in many ways.

Computer resources used to produce this paper were made available by Pinetree Management, Inc.

\section{References}

Day, R. H. (1997) Complex Dynamics, Market Mediation and Stock Price Behavior. North American Actuarial Journal, 1, $6-23$.

Edwards, R. D. and Magee, J. (1992) Technical Analysis of Stock Trends. John Magee, New York.

O'Neil, W. J. (1995) How to Make Money in Stocks. McGrawHill, New York.

Pring, M. J. (1991) Technical Analysis Explained. McGraw-Hill, New York, p. 186.

Ross, D. M. (1998) The Ross Multiple Moving Averages Method - Using Separate Moving Averages for Buy \& Sell Signals in Equity Markets. Market Technicians Association Journal, 50, $31-47$.

Wang, J. K. (1998) Discussion on 'Complex Dynamics, Niarket Mediation and Stock Price Behavior,' by Richard H. Day. North American Actuarial Journal, 2, 117-118.

Wang, J. K. (preprint, 2001) 'Behavioral Econometric' Interpretation of Dynamic Supply and Demand Functions in a Market Pricing Model. To be published in International Journal of Theoretical and Applied Finance, 4(3). 


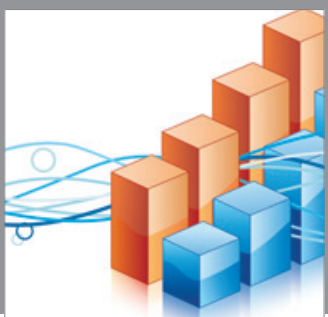

Advances in

Operations Research

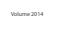

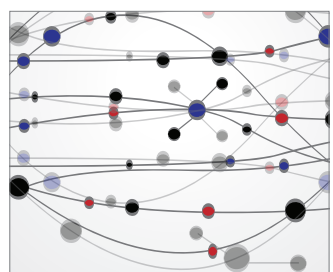

\section{The Scientific} World Journal
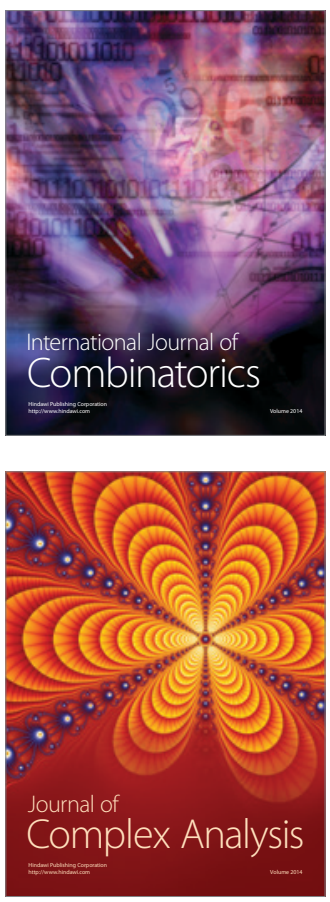

International Journal of

Mathematics and

Mathematical

Sciences
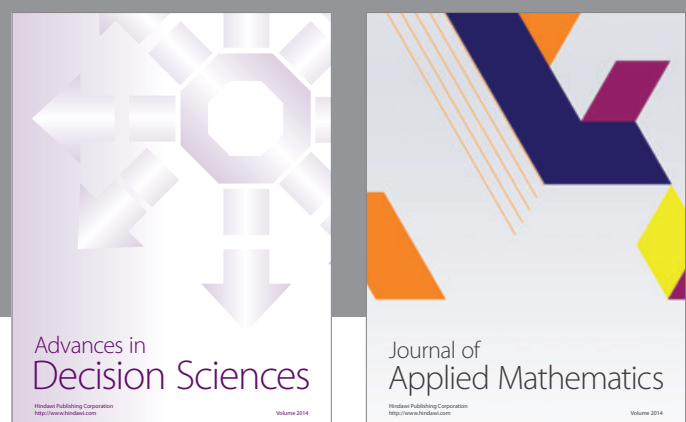

Journal of

Applied Mathematics
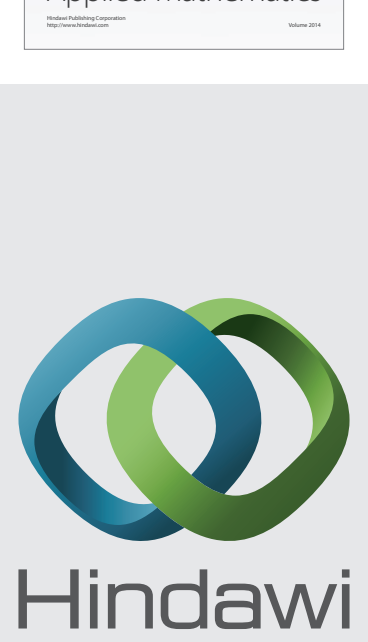

Submit your manuscripts at http://www.hindawi.com
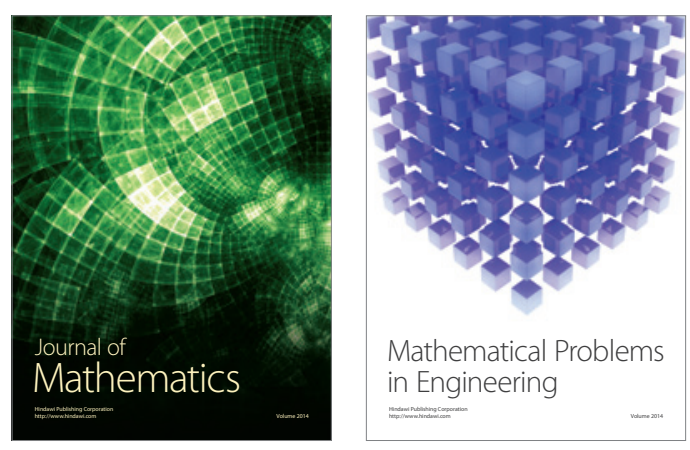

Mathematical Problems in Engineering
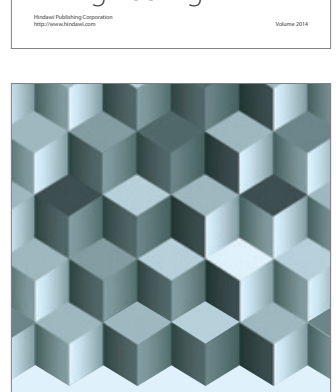

Journal of

Function Spaces
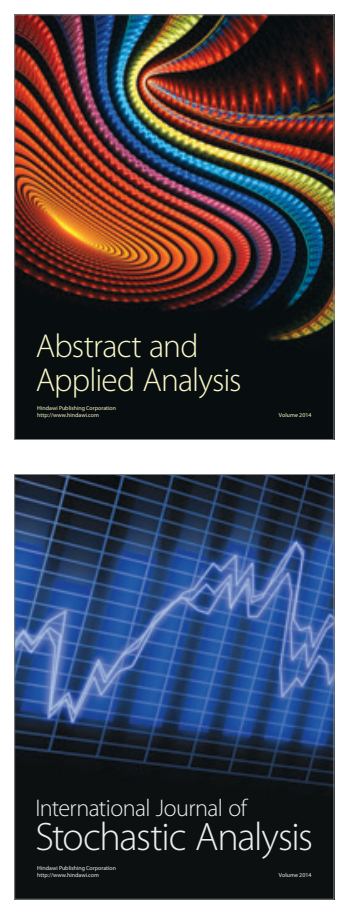

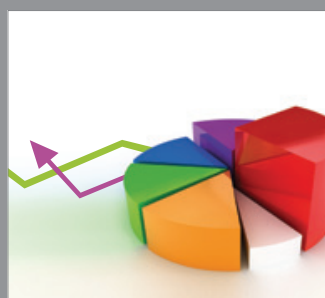

ournal of

Probability and Statistics

Promensencen
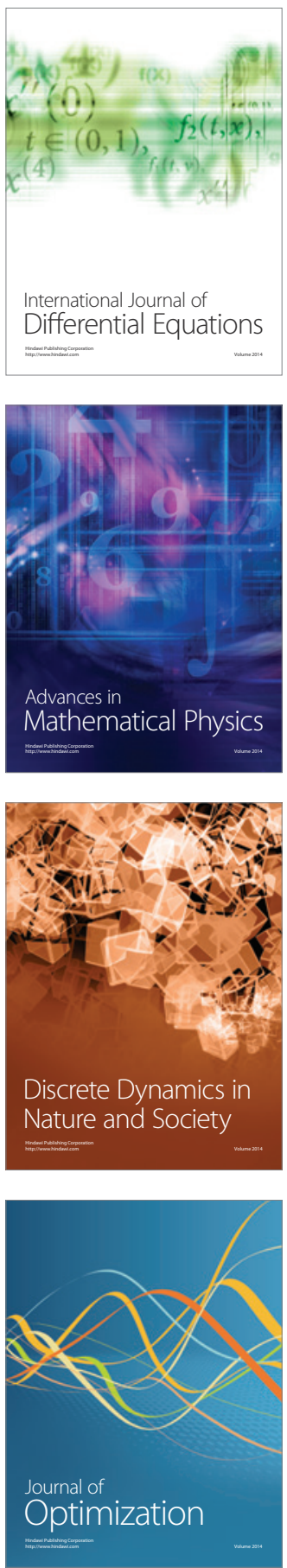